\title{
Friction Laws Determination of Random-Textured Surface of Microcrystalline Diamond Coatings, Using the Oscillating Tribometer Technique
}

\author{
Michel Belin ${ }^{1)^{*}}$, Hiroyuki Miki ${ }^{2)}$ and Toshiyuki Takagi ${ }^{3)}$ \\ ${ }^{1)}$ Laboratoire de Tribologie et Dynamique des Systèmes, UMR 5513, \\ École Centrale de Lyon, Université de Lyon, Écully cedex F69134, France \\ ${ }^{2)}$ Frontier Research Institute for Interdisciplinary Sciences, Tohoku University, \\ 6-3, Aramaki Aza Aoba, Aoba-ku, Sendai, Miyagi 980-8578, Japan \\ ${ }^{3)}$ Institute of Fluid Science, Tohoku University, \\ 2-1-1, Katahira, Aoba-ku, Sendai, Miyagi 980-8577, Japan \\ *Corresponding author: Michel Belin (michel.belin@ec-lyon.fr)
}

Manuscript received 06 November 2018; accepted 06 June 2019; published 15 July 2019

\begin{abstract}
Achieving low-friction and robust tribosystems is an open and challenging question to tribologists. In this study, we are considering the diamond films as candidates. Quantification of friction is obtained here using an original technique, so called the oscillating relaxation tribometry. It is based on the study of the oscillating motion of a free-damped 1-DOF mechanical oscillator, the sliding contact acting as a main source of mechanical dissipation ("relaxation"). Therefore, it is possible to determine friction value and its sliding speed dependency, i.e. a kind of "Stribeck curve" under non-stationary conditions. The interest of such "relaxation tribometer" is to characterize low friction with an unequalled sensitivity, and to directly obtain the "friction law" thanks to a robust and rapid experimental test. Polycrystalline diamond films are deposited by the Hot Filament CVD method on SiC substrate, which were partly polished and finished to gradient surface roughness with random-textured asperities. Stainless steel balls of $6.0 \mathrm{~mm}$ diameter are used as the rubbing counterface. The normal force is varied between 50 and $300 \mathrm{mN}$, leading respectively to a maximum Hertzian contact pressure of 0.30 and $0.71 \mathrm{GPa}$, respectively. Taking into account the experimental setup, the sliding speed is decaying as a damped pseudo-sine motion, the maximum sliding value being set to $0.16 \mathrm{~m} / \mathrm{s}$, progressively decaying to 0 when mechanical equilibrium is reached, within a typical duration of $5 \mathrm{~s}$. First results are reported here, using this innovative friction characterization technique. The different contributions to friction of the surface roughness are analyzed and discussed. These results show the major interest of the use of this method to characterize the low-friction behavior of textured diamond films. These results are discussed and the high potential of such textured films in actual tribosystems is presented here.
\end{abstract}

\section{Keywords}

friction, damping, relaxation tribometer, transient oscillations, diamond films, textured surfaces

\section{Introduction}

The low friction tribosystems is one of the main issues in different fields of modern industry, like microelectronics, food and aerospace industries. Some answers to this problem are carbon-based materials like diamond-like carbon (DLC) and diamond. In addition, the debris reduction is required to prevent pollution and malfunction. In such case, diamonds films have remarkable characteristics, in terms of tribological behavior and chemical inertness. Especially, the flat and smooth surface of diamond shows excellent tribological performance $[1,2]$.

Diamond is well known for its excellent properties such as high hardness, low friction, good thermal conductivity and wide band gap structure [3]. Although a natural diamond is extremely precious and expensive, techniques for artificial diamond manufacture, such as the chemical vapor deposition method in which gas is used as the raw material, have been developed. In many cases, artificial diamond is a polycrystalline structure and its surface is composed of small crystalline diamond and the surface shape of polycrystalline diamond film is rough, much like the surface of a whetstone. High surface roughness is essentially undesirable for many practical applications [4]. Reducing the surface roughness of polycrystalline diamond films is a critical requirement for its application in wear-resistant, low friction, coatings [5]. The effect of surface roughness on friction phenomena is inconclusive, but surface roughness is considered one of the major influences in tribological behavior. 
Technically speaking, it is very difficult to control arbitrary surface roughness of polycrystalline diamond films because of its high hardness and difficulty in processing. To overcome this problem we have developed easily abradable diamond films in previous work [6]. The diamond films investigated can be categorized as Ballas diamond, first discovered by Fischer [7]. Ballas diamonds are also called 'cauliflower-like' and 'ball-shaped' by some authors. Much research has been conducted into Ballas type diamond films [8-10]. These mainly consist of fine crystal diamond with random orientation. These polycrystalline diamonds are connected to each other in an amorphous carbon matrix, which makes an easy to polish diamond surface $[6,11]$.

In previous research, Miki et al. investigated the friction properties of diamond-metal surfaces with the aim of producing practical uses for polycrystalline diamond films [11]. A low coefficient of friction $\mu=0.08$ was achieved for diamond film polished to $R \mathrm{a}=0.05 \mu \mathrm{m}$, where $R \mathrm{a}$ is the arithmetic average surface roughness.

In addition, partially polished diamond film is expected to be applied for solid lubricant film because it has good frictional properties at high sliding speed without lubricant oil. Nakamori et al. [1] and Yonemura et al. [2] described that the drop-off of coefficient of friction (CoF) on diamond film is caused by "lift-off" due to increase of lift force generated by the pressure change between partially polished diamond film and counter material. In addition, Miki et al. [12, 13] found that $\mathrm{CoF}$ and drop-off threshold on CoF of partially polished diamond film is reduced as arithmetic average surface roughness $(\mathrm{Ra})$ of the film decreases. However, the detailed relationship between the surface geometry of polished diamond film and lift-off phenomenon is still unclear.

Nevertheless, quantification of friction is made difficult when the value of friction is reached in the order of $10^{-3}$ or lower. To solve this problem, we have developed a novel friction measurement technique based on the "relaxation" method, which allows the determination of friction without any friction force measurement.

We present here some results obtained using the "relaxation" tribometer, giving access to the specific friction law, and so bringing a new insight on friction behavior of tribosystems.

\section{Experimental}

2.1 Preparation of diamond films In this experiment, diamond film was deposited by a hot- filament chemical vapor deposition (HFCVD) method. HFCVD is the method that excites hydrocarbon gas by electrical heating of filaments to deposit carbon elements onto a substrate. We used tungsten filament with a diameter of $0.3 \mathrm{~mm}$. Deposition parameter of HFCVD process is shown in Table 1. The substrate is $\mathrm{SiC}$ disk $(\phi 50 \mathrm{~mm} \times 3 \mathrm{~mm})$. Before the deposition, seeding treatment was done with diamond particle. The details of the deposition and pre-processing of the substrate are described in somewhere [13].

After the deposition, the diamond film was polished up to 3 zone as follows, -zone 1: Ra from 0.1 to $0.2 \mu \mathrm{m}$, -zone 2: Ra from 0.4 to $0.7 \mu \mathrm{m}$ and -zone 3: Ra from 1.0 to $1.5 \mu \mathrm{m}$ respectively. By this polishing, surface of the diamond film became the partly flat shape. Typical plane view of laser microscope and 3D topographic image of the surface of polished diamond film showing the basic shape of multiple plateaus and valleys were obtained by using the 3D Laser Scanning Confocal Microscope (VK-X200, KEYENCE) in Figs. 1 and 2 respectively.

Table 1 Deposition parameter of HFCVD for polycrystalline diamond

\begin{tabular}{|c|c|}
\hline $\mathrm{H}_{2}$ flow & $100 \mathrm{ml} / \mathrm{min}$ \\
\hline $\mathrm{CH}_{4}$ flow & $2.3 \mathrm{ml} / \mathrm{min}$ \\
\hline Total gas pressure & $8.0 \mathrm{kPa}$ \\
\hline Deposition time & 5 hours \\
\hline Filament temperature & $2000 \mathrm{~K}$ \\
\hline
\end{tabular}

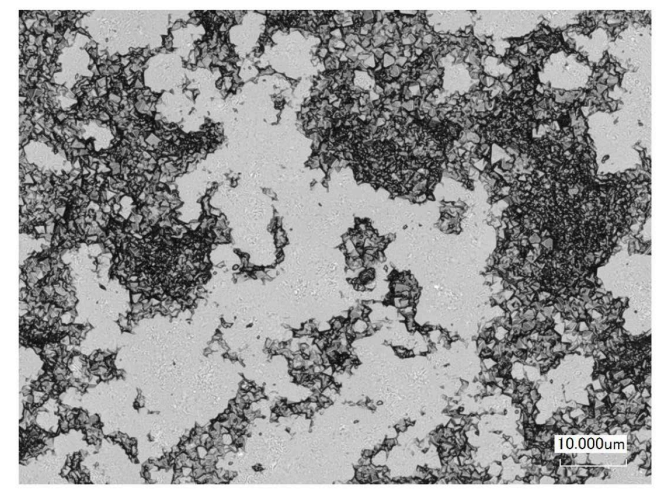

Fig. 1 Typical Laser microscope image of partially polished diamond film: Zone $1(R a=0.11 \mu \mathrm{m})$. We can observe the plateaus of the resulting topography due to partial polishing.

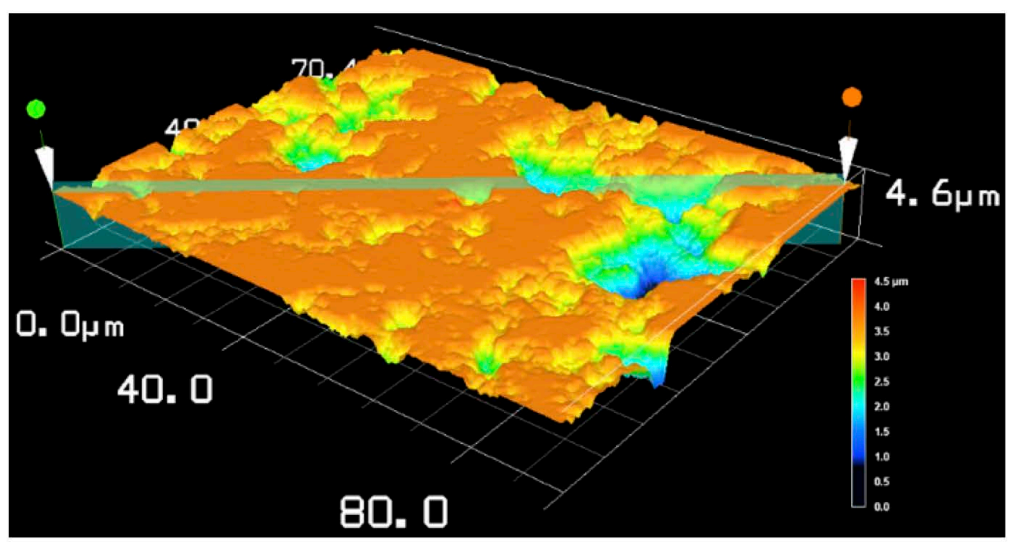

Fig. 2 Typical 3D topographic image of a partially polished diamond film: Zone $1(R a=0.11 \mu \mathrm{m})$. We confirm the presence of plateaus, separated by initial grooves and valleys due to the deposition process. 
2.2 Testing with the relaxation tribometer

The "relaxation" method consists in evaluating friction without direct measurement of the tangential force. Actually, the friction measurement is based on the analysis of the freeresponse velocity of a spherical pin involved in a sphere-onplane contact. The spherical pin is connected to a support mounted on a double leaf elastic spring, here called bi-blades, and the plane is fixed. The mechanical system principle is depicted in Fig. 3. It consists in a single degree-of-freedom (1DOF) mechanical oscillator, damped by the rubbing contact. The steel elastic bi-blade allows the spherical pin to oscillate horizontally from an out-of-equilibrium initial position imposed thanks to an electromagnet. In other words, the electromagnet is used to initiate the movement and when it is deactivated, the spherical pin oscillates freely on the sample until the stop induced by energy dissipation due to the friction force. Normal load is applied and remains constant during the oscillatory test, the spherical pin moving parallel to the plane, until stop. The velocity time-response is measured using a laser velocimeter.

The analysis is based on the study of the oscillating motion of a free-damped 1-DOF mechanical oscillator, the sliding contact acting as a main source of mechanical dissipation ("relaxation"). Therefore, it is possible to determine friction value and its sliding speed dependency, under non-stationary conditions. The interest of such "relaxation tribometer" is to characterize low friction with an unequalled sensitivity, and to directly obtain the "friction law" thanks to a robust and rapid experimental test. Taking into account the experimental setup, the sliding speed is decaying as a damped pseudo-sine motion, the maximum sliding value being set to $160 \mathrm{~mm} / \mathrm{s}$, progressively decaying to 0 when mechanical equilibrium is reached, within a typical duration of some seconds.

\subsection{Identification of the friction law}

In previous studies [14, 15], the authors have reported how the relaxation tribometer technique was able to discriminate different contributions to friction: velocity-dependent and velocity-independent. Another result shows that the friction evolution depends on the velocity amplitude of the free oscillating response. On this basis, Majdoub et al. [16] propose to determine the friction coefficient from the free-damped response of the sliding 1-DOF oscillator.

In the present study, we introduce a very simple way to do that by assuming a constant friction coefficient at each oscillation. Thus, consider the equation motion written as follows:

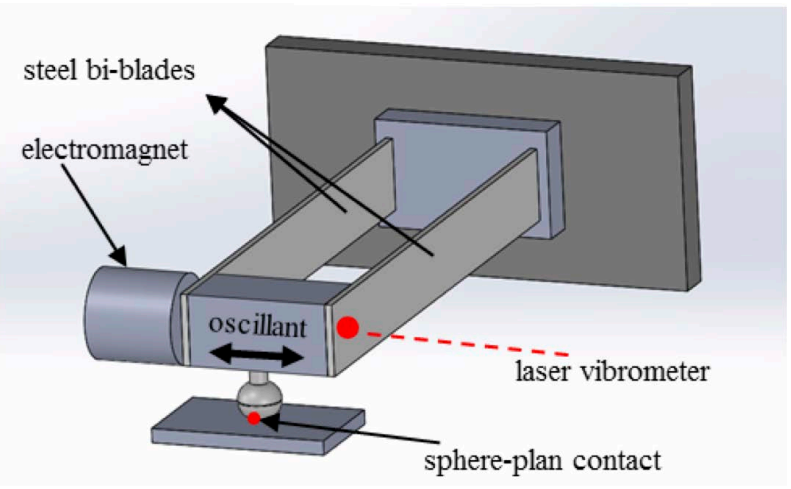

Fig. 3 Schematic of the relaxation oscillating tribometer

$$
m \ddot{x}+k x= \pm \mu N
$$

where $x$ is the displacement, $\ddot{x}$ is the corresponding acceleration, $m$ is the moving mass and $k$ the spring stiffness. $N$ is the applied normal load and $\mu$ is the kinematic friction coefficient to be determined. From this decaying amplitude of the oscillations, we can extract friction coefficient in a classical way.

As described in detail in a joint paper [17], the analysis of this response of a damped 1-DOF system is well known and shows that the decay between three successive peaks of the maximum velocity is related to the friction coefficient $\mu$ by the following relation, where $\dot{x}$ is the sliding velocity:

$$
\mu\left(V_{\max }\right)=\Delta \dot{x}_{3 p e a k s} \frac{\sqrt{k m}}{8 N}, N \neq 0
$$

Therefore, by plotting the evolution $\mu=f\left(V_{\max }\right)$, we can access the evolution of the friction value with sliding velocity, in nonstationary conditions. We call that the "instantaneous friction law". We can qualitatively describe the friction process with sliding velocity. We can also quantify the two contributions to friction that we consider in thjs paper: the "Coulomb type" one (sometimes called "solid friction"), and the "viscous-type" one.

\section{Operating conditions and results}

The experiment reported in this study consists on a dry contact between the diamond sample and a steel ball, AISI 52100 , diameter equal to $6.0 \mathrm{~mm}$. The experiments were run in ambient air $(\mathrm{RH}=38 \%)$ at room temperature $\left(23^{\circ} \mathrm{C}\right)$. A normal load ranging from 50 to $300 \mathrm{mN}$ was applied, leading to an initial maximum contact pressure ranging from 300 to 540 MPa. In the relaxation mode, the maximum value of speed $V_{\max }$ was $160 \mathrm{~mm} / \mathrm{s}$ and a duration of 2 to 20 seconds was chosen to record the time-response of the velocity. Each experiment in the relaxation mode was reproduced three times to check the good repeatability of the oscillating time responses.

\subsection{Intrinsic damping of the device itself}

We first run relaxation tests without any contact or normal load, to check the damping behavior of the machine itself. Figure 4 shows the results: Fig. 4(a) in blue: the time response of the velocity, Fig. 4(a) in red: the envelope of velocity maxima $V_{\max }$ and Fig. $4(\mathrm{~b})$ the friction coefficient, computed from the envelope. We find that the damping of the testing equipment is velocity dependent, with a purely "viscous-type" behavior. No "Coulomb" contribution is observed.

\subsection{Response of the diamond film}

We are now considering a contact between the diamond sample and a sphere steel ball, loaded by a given normal force. Three zones, with three different roughness are tested-zone 1: Ra from 0.1 to $0.2 \mu \mathrm{m}$, -zone 2: Ra from 0.4 to $0.7 \mu \mathrm{m}$ and -zone 3: 1.0 to $1.5 \mu \mathrm{m}$. We present results obtained with a normal load value ranging from 50 to $300 \mathrm{mN}$, see Fig. 5 . We find that:

- The number of oscillations needed to reach stop is strongly dependent on the normal load, as expected,

- And also, the smaller the roughness, the lower the damping.

We focus on the comparison between results obtained with a normal load value of $50 \mathrm{mN}$, Fig. 6(a)-(c). We find that:

- The damping is strongly dependent on the surface roughness, the lower roughness, the lower damping;

- The friction values are ranging from 0.4 down to 0.08 for 
(a)

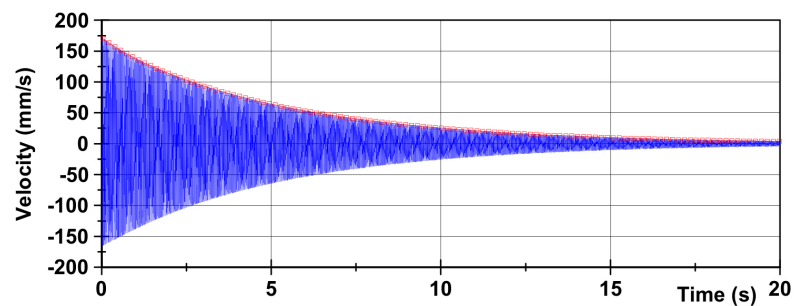

(b)

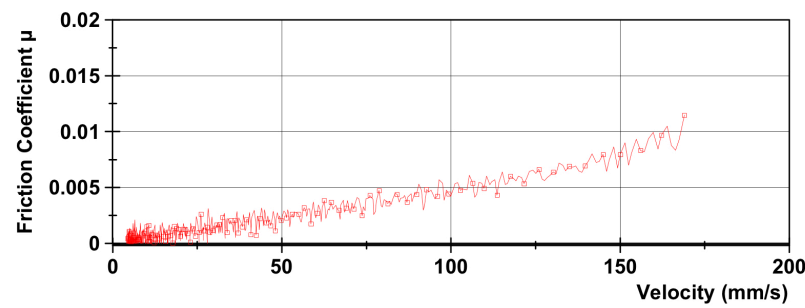

Fig. 4 Damping characterization of the relaxation tribometer, in free relaxation, no contact, (a) In blue: the time response of the velocity, dots in red: the envelope of the maximum velocity $V_{\max }$ (b) The red line: the computed friction coefficient versus maximum speed $V_{\max }$. In this case, we only detect a linear viscous-type contribution to friction.

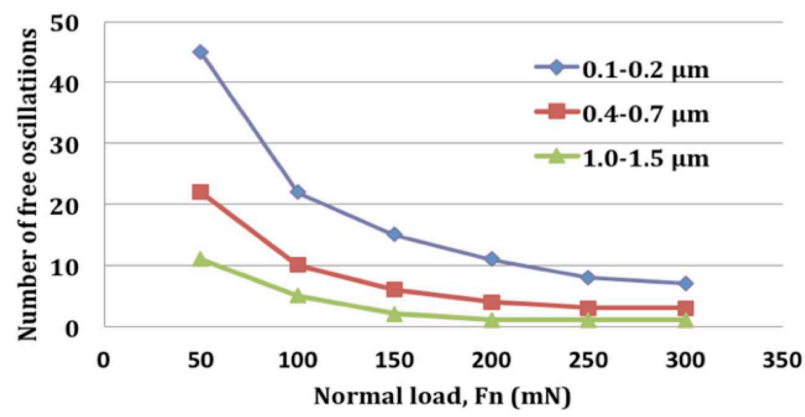

Fig. 5 Evolution of the number of free damped oscillations, obtained with diamond coatings, for different topography. Roughness Ra: 0.1-0.2 $\mu \mathrm{m}, 0.4-0.7 \mu \mathrm{m}, 1.0-1.5 \mu \mathrm{m}$

the smaller roughness zone;

- The "friction law", i.e. $\mu=f\left(V_{\max }\right)$ is a combination of Coulomb-type contribution and a linear viscous-type one.

For a better description of results, we have plotted the different "friction laws" obtained with the different roughness values, on a same graph, Fig. 7 . Friction is considered in the form of:

$$
\mu=\mu_{0}+\mu_{1} \cdot V_{\max }
$$

We get $\mu_{0}$ value by extrapolating the friction law to $V_{\max }=0$. The value $\mu_{1}$ is the slope of the friction law. The values are reported in Table 2. L So we can quantify the two contributions to friction. We observe the strong decrease of the friction value when the roughness is decreased.

Let us consider now only the low roughness zone (zone 1), tested with different normal loads: 50, 100 and $200 \mathrm{mN}$. The results are reported in Table 3.

We observe that:

- the friction laws are superimposed for the 3 cases of (a)
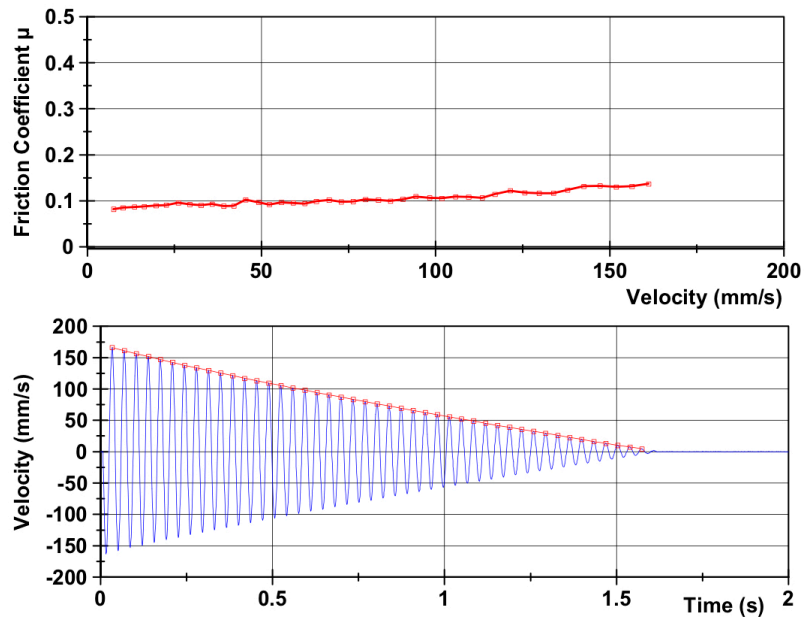

(b)
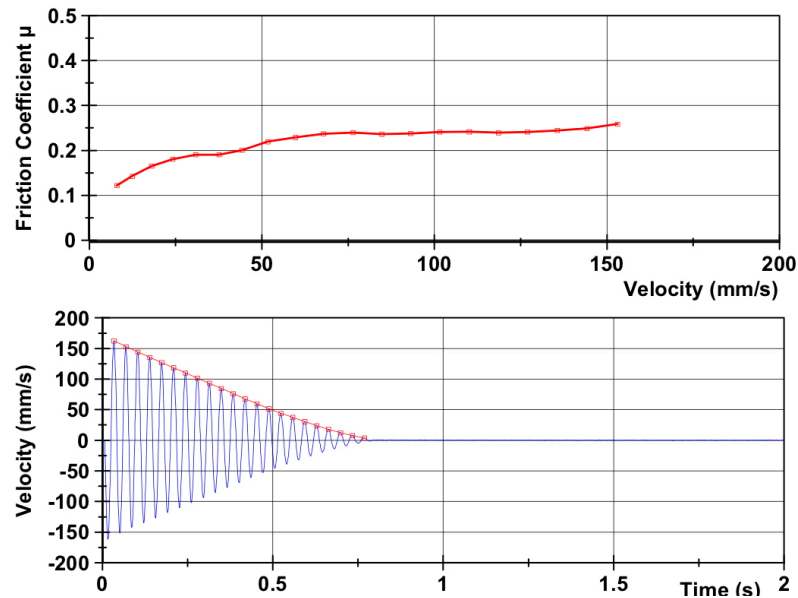

(c)
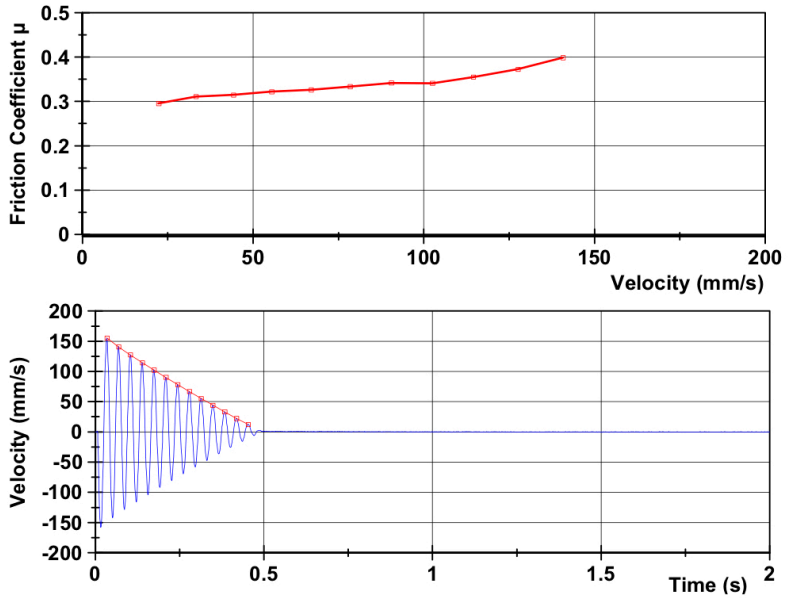

Fig. 6 Typical relaxation results, obtained on the 3 different roughness zones. In blue, the velocity time-response of the test, dots in red: the detected envelope of the velocity maxima, and full line in red: the computed friction law, (a) Zone 1: Ra: 0.1 to $0.2 \mu \mathrm{m}$, (b) Zone 2: Ra: 0.4 to $0.7 \mu \mathrm{m}$, (c) Zone 3: Ra: 1.0 to $1.5 \mu \mathrm{m}$ 
Table 2 Friction values extracted from the friction laws shown Fig. 7. We can discriminate the solid friction contribution (dimensionless) and the viscous-type one (dimension: s.m-1).

\begin{tabular}{|c|c|c|c|}
\hline $\mathrm{N}=50 \mathrm{mN}$ & & Coulomb contribution & "Viscous-type "contribution \\
\hline & $R \mathrm{a}(\mu \mathrm{m})$ & $\mu_{0}$ (dimensionless) & $\mu_{1}\left(\mathrm{~s} . \mathrm{m}^{-1}\right)$ \\
\hline Zone 1 & $0.1-0.2$ & 0.08 & 0.2 \\
\hline Zone 2 & $0.4-0.7$ & $/$ & $/$ \\
\hline Zone 3 & $1.0-1.5$ & 0.28 & 0.9 \\
\hline
\end{tabular}

Table 3 Friction values extracted from friction laws when the normal load is varied. We show a good stability of both $\mu_{0}$ and $\mu_{1}$ values.

\begin{tabular}{|c|c|c|c|}
\hline Zone 1 & $\begin{array}{c}\text { Coulomb } \\
\text { contribution }\end{array}$ & $\begin{array}{c}\text { "Viscous-type" } \\
\text { contribution }\end{array}$ \\
\hline Ra $(\mu \mathrm{m})$ & $\mathrm{N}(\mathrm{mN})$ & $\begin{array}{c}\mu_{0} \\
\text { (dimensionless) }\end{array}$ & $\begin{array}{c}\mu_{1} \\
\left(\mathrm{~s}^{-1} \mathrm{~m}^{-1}\right.\end{array}$ \\
\hline $0.1-0.2$ & 50 & 0.08 & 0.2 \\
\hline $0.1-0.2$ & 100 & 0.08 & 0.2 \\
\hline $0.1-0.2$ & 200 & 0.08 & 0.2 \\
\hline
\end{tabular}

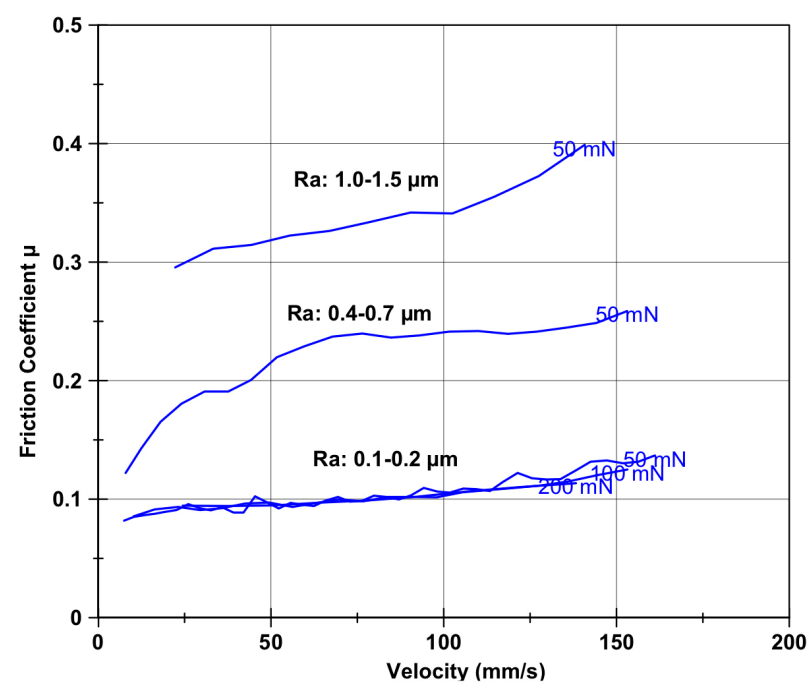

Fig. 7 Friction laws obtained on the partially polished diamond film, for the different roughness values. In each case, we find a combination of "viscous-type" and "Coulomb-type" contributions. As expected, the larger the roughness value, the larger the Coulomb friction contribution.

normal load (Fig. 7). So, the calculated value of $\mu_{0}$ and $\mu_{1}$ are the same (Table 3). These results give evidence for a major contribution of "Coulomb-type" friction, with a small contribution of "viscous-type" one;

- the total friction is ranging from 0.08 to 0.14 , when maximum velocity varies from 0 to $0.16 \mathrm{~m} / \mathrm{s}$. These values are fairly low, and can be interesting for some applications in practical tribosystems, where moderate friction and no wear is required.

In this method, the "friction law" that is computed from relaxation data gives us a general trend,

- for data at low- and high-roughness, the situation is clear. The Coulomb-type value $\mu_{0}$ can be defined as the extrapolation of the friction law, when velocity tends to zero value,

- for the medium-roughness case, the tendency is not clear, showing a kind of inflexion point. Therefore, the determination of $\mu_{0}$ is difficult. The reason for that remains unclear at this stage. Investigation is still in progress.

The characterization technique as presented here is rapid and robust. We can notice that lower friction values have been reported in literature during tests at lower contact pressures [13]. The potential role of lubrication by an air film has to be clarified.

\section{Conclusions}

First results are reported here, using this innovative friction characterization technique. The different contributions to friction of the surface roughness are analyzed and discussed. These results show the major interest of the use of this method to characterize the low-friction behavior of textured diamond films. It is shown that the friction value strongly depends on the roughness of the sample, as expected. The results show that the friction behavior is essentially "Coulomb-type", with a slight contribution of "viscous-type" friction.

These results show the high potential of such textured films in actual tribosystems were controlled friction and clean environment are required.

\section{Acknowledgements}

The authors are grateful to CNRS and the International Collaborative Research Project of Frontier Research Institute for Interdisciplinary Sciences, Tohoku University, for supporting exchanges in the frame of ELyT Lab, that make this work possible. They also want to acknowledge Kyohei Naito for preparing the samples and Nathan Gerbi for precious help in relaxation data processing.

\section{References}

[1] Nakamori, I., Takagi, T., Takeno, T., Abe, T., Uchimoto, T. and Kohama, Y., "Direct Simulation of Monte Carlo Analysis of NanoFloating Effect on Diamond-Coated Surface," Diamond and Related Materials, 14, 11-12, 2005, 2122-2126.

[2] Yonemura, S., Yamaguchi, M., Takeno, T., Miki, H. and Takagi, T., "Effect of Micro Gas Flow on Low Friction Properties of Diamond Coating with Partly Polished Surface," AIP Conference Proceedings, 1084, 1, 2008, 1153-1157.

[3] Yoshikawa, M. and Otake, N., "Chemical Vapor Deposition Diamond," Ohmsha, Ltd., 1995 (in Japanese).

[4] Moseler, M., Gumbsch, P., Casiraghi, C., Ferrari, A. C. and Robertson, J., "The Ultrasmoothness of Diamond-Like Carbon Surfaces," Science, 309, 5740, 2005, 1545-1548.

[5] Spear, K. E. and Dismukes, J. P. (Eds.), "Synthetic Diamond: 
Emerging CVD Science and Technology," John Wiley and Sons, Inc., USA, 1993.

[6] Abe, T., Takagi, T., Sun, Z. M., Uchimoto, T., Makino, J. and Hashimoto, H., "Machinable Ceramic Substrate for CVD Diamond Coating," Diamond and Related Materials, 13, 4-8, 2004, 819-822.

[7] Fischer, R. B., "The 'Ballas' Form of Diamond," Nature, 189, 1961 50.

[8] Huang, B. R., Chia, C. T., Chang, M. C. and Cheng, C. L., "Bias Effects on Large Area Polycrystalline Diamond Films Synthesized by the Bias Enhanced Growth Technique," Diamond and Related Materials, 12, 1, 2003, 26-32.

[9] Yang, T. S., Lai, J. Y., Cheng, C. L. and Wong, M. S., "Growth of Faceted, Ballas-Like and Nanocrystalline Diamond Films Deposited in $\mathrm{CH}_{4} / \mathrm{H}_{2} / \mathrm{Ar}$ MPCVD," Diamond and Related Materials, 10, 12, 2001, 2161-2166.

[10] Moriyoshi, Y., Kamo, M., Setaka, N. and Sato, Y., “The Microstructure of Natural Polycrystal Diamond, Carbonado and Ballas," Journal of Materials Science, 18, 1, 1983, 217-224.

[11] Takeno, T., Komoriya, T., Nakamori, I., Miki, H., Abe, T., Uchimoto, T. and Takagi, T., "Tribological Properties of Partly Polished Diamond Coatings," Diamond and Related Materials, 14, 11-12, 2005, 2118-2121.

[12] Miki, H., Yoshida, N., Bando, K., Takeno, T., Abe, T. and Takagi, T.,
"Atmosphere Dependence of the Frictional Wearing Properties of Partly-Polished Polycrystalline Diamonds," Diamond and Related Materials, 17, 4-5, 2008, 868-872.

[13] Miki, H., Tsutsui, A., Takeno, T. and Takagi, T., "Friction Properties of Partially Polished CVD Diamond Films at Different Sliding Speeds," Diamond and Related Materials, 24, 2012, 167-170.

[14] Majdoub, F., Belin, M., Martin, J. M., Perret-Liaudet, J., Kano, M. and Yoshida, K. "Exploring Low Friction of Lubricated DLC Coatings in No-Wear Conditions with a New Relaxation Tribometer," Tribology International, 65, 2013, 278-285.

[15] Belin, M., Kakizawa, M., Rigaud, E. and Martin, J. M., “Dual Characterization of Boundary Friction Thanks to the Harmonic Tribometer: Identification of Viscous and Solid Friction Contributions," Journal of Physics: Conference Series, 258, 1, 2010, 012008.

[16] Majdoub, F., Perret-Liaudet, J., Belin, M. and Martin, J. M., “Decaying Law for the Free Oscillating Response with a Pseudo-Polynomial Friction Law: Analysis of a Superlow Lubricated Friction Test," Journal of Sound and Vibration, 348, 2015, 263-281.

[17] Hriouech, A., Belin, M., Perret-Liaudet, J., De Barros M. I. and Diaby, M., "An Original Tribometer Coupling a Free-Oscillation Dynamic Tribotest with a Conventional Linear Reciprocating Tribometer," Proc. WTC 2017, Beijing, 2017, Poster ST-A-019. 